\title{
Doppler Compensation Based on Recursive Block for Wireless Communication
}

\author{
Chan-Ho Hwang and Ki-Man Kim
}

\begin{abstract}
The Doppler effect and fading that has time variance affect the wireless communication reliability. Especially, the Doppler effect reduces the transmission efficiency. Thus, we need to recognize the channel state and apply it to communication technique for increasing transmission efficiency. In this paper, we present the frame recursive modulation and demodulation method using ambiguity function and Kay's estimator to estimate the Doppler frequency. Furthermore, we conducted the simulation in environment with Rayleigh fading and the Doppler effect. When the channel coding technique was not used, the bit error rate of the proposed method was improved maximum $17 \%$ compared with conventional method.
\end{abstract}

Index Terms-Doppler frequency estimation, ambiguity function, Kay's estimator, Rayleigh fading.

\section{INTRODUCTION}

The development of public transportation and communications revolutionized the way people communicate. The age of high capacity and high speed wireless communication has come. Accordingly, the communication between moving subjects like satellite has been essential and the Doppler effect by movement has been important element in wireless communication [1], [2]. The Doppler affects the temporal coherence related transmission efficiency [3]. The transmission efficiency is the ratio between the actually demodulated data packet length and total communication frame length and depends on channel variance. Therefore, we need to compensate for the Doppler frequency in order to increase the transmission efficiency. In practical, there are several Doppler estimation techniques. The simplest method is based on the fast Fourier transformation. Other methods are ambiguity function, block Doppler estimation and interpolation method. These techniques are used for underwater acoustic communication as well as radio communication [4].

In this paper, we present the two Doppler shift frequency estimation techniques and propose the frame recursive modulation and demodulation method which can estimate the channel parameter during the data frame using the presented Doppler estimation techniques. Simulation was performed to compare the performance of the proposed method with a

Manuscript received October 15, 2014; revised June 12, 2015. This work was supported by Ministry of Education and National Research Foundation of Korea (NRF) through the Human Resource Training Project for Regional Innovation and by Agency for Defense Development under the Contract UD130007DD.

The authors are with the Department of Radio Communication Engineering, Korea Maritime and Ocean University, Busan, Republic of Korea (e-mail: hwangchanho@kmou.ac.kr). conventional non-recursive Doppler estimation.

\section{DOPPLER ESTIMATION TECHNIQUES}

\section{A. Ambiguity Function Method}

The Doppler shift frequency is defined as the ratio between signal propagation speed and the source relative velocity [5]. When the Doppler shift frequency is $\Delta$, the signal with Doppler effect can be expressed as follows:

$$
r(t)=s((1+\Delta) t)
$$

where $r(t)$ is the received signal, and the transmitted signal is $s(t)$. The ambiguity function is same as the matched filter response against Doppler shift value and delay of the incoming signal [6]. For a continuous time signal with wide band, the ambiguity function is given as:

$$
A_{s}(\tau, \Delta)=(1+\Delta) \int_{-\infty}^{\infty} s((1+\Delta) t) s(t-\tau) d t
$$

$\tau$ is the delay and $\Delta$ is the Doppler shift frequency. If the received signal $r(t)$ is considered, we can express the cross-ambiguity function:

$$
A_{s r}(\tau, \Delta)=(1+\Delta) \int_{-\infty}^{\infty} s((1+\Delta) t) r(t-\tau) d t
$$

To estimate the Doppler shift, we have to search in $\Delta$ with time alignment and find the maximum value of the cross-ambiguity function:

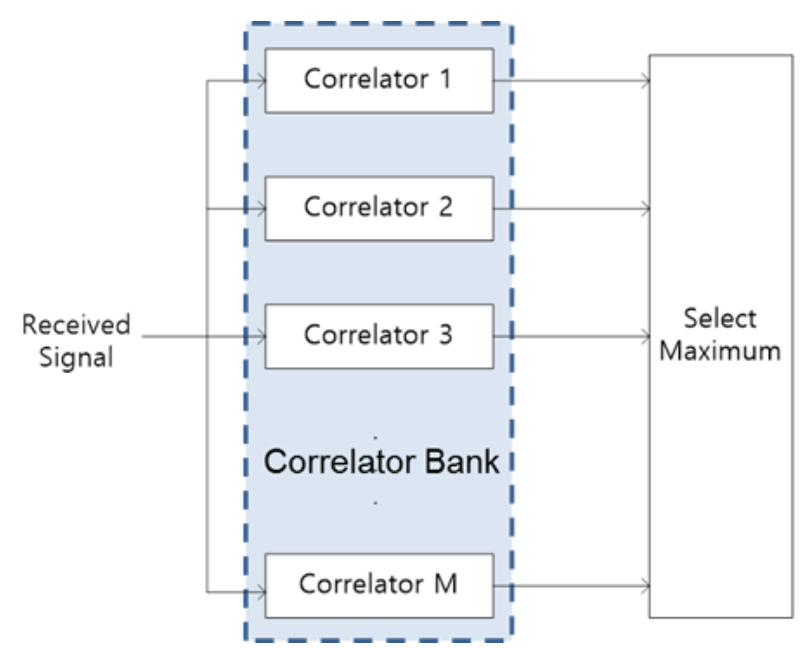

Fig. 1. Structure of correlator-banks. 


$$
A_{s r}(0, \Delta)=(1+\Delta) \int_{-\infty}^{\infty} s((1+\Delta) t) r(t) d t
$$

When the value of (4) is maximized, $\Delta$ is estimated as the Doppler shift in the received signal. In practice, we conduct the correlation between received signal and Doppler-shifted replicas which consist of correlator-banks. There is constant frequency interval among the each replica. Fig. 1 shows the structure of correlator-banks.

\section{B. Kay's Estimator}

If the modulation is PSK (phase shift keying) with perfect timing synchronization, the Doppler frequency can be estimated by using the auto-correlation [7]. In the training mode, the received signal after matched filtering is given by

$$
x(k)=a_{k} e^{j(2 \pi \Delta f k T+\theta)}+n(k)
$$

$a_{k}$ is $k$-th symbol amplitude, $\Delta f$ is the Doppler shift frequency, $\mathrm{T}$ is the symbol period, $\theta$ is the carrier phase, and $n(k)$ is the white Gaussian noise with zero mean. In the training mode, modulation can be easily removed by multiplying $x(k)$ with $a_{k}^{*}$ and $*$ means complex conjugate.

$$
z(k)=a_{k}^{*} a_{k} e^{j(2 \pi \Delta f k T+\theta)}+a_{k}^{*} n(k)
$$

$z(k)$ can be considered as a complex sinusoid superimposed on white Gaussian noise. Using (6), multiplying $z(k)$ by $z^{*}(k-1)$ can be expressed as follow:

$$
\begin{aligned}
R(k) & =z_{k}(k) \times z_{k}^{*}(k-1) \\
& =e^{j 2 \pi \Delta f k T}\left(1+\tilde{n}(k)+\tilde{n}^{*}(k-1)+\tilde{n}(k) \times \tilde{n}^{*}(k-1)\right)
\end{aligned}
$$

Assume the SNR (signal-to-noise ratio) is enough, the Doppler shift frequency can be taken by the argument of (7). The $\arg ()$ means the phase.

$$
\Delta \hat{f}=\frac{1}{2 \pi T} \arg \left(z_{k}(k) \times z_{k}^{*}(k-1)\right)
$$

\section{DOPPLER FREQUENCY ESTIMATION BY FRAME RECURSIVE MODULATION AND DEMODULATION METHOD}

\section{A. Recursive Frame}

In general, the communication data packet consists of training section and data frame section [8]-[10]. The training mode which the transmitter and the receiver are already known is used to update the tap coefficients for operating the equalizer and to estimate the Doppler frequency. However, the estimated values which affect the communication performance are changed by time variance of the channel during the data frame. These variances reduce the communication reliability. Therefore, we need to estimate and to compensate the Doppler frequency in data frame. In conventional communication method, the data frame is only demodulated using the estimated channel parameter in the training mode. However, the proposed frame recursive modulation and demodulation method is able to estimate the varying channel parameter during the data frame and has the advantage in terms of communication performance.

The received data packet is divided into several data frame called recursive data frame. First recursive data frame is demodulated using the channel parameter of the training mode. Then, the demodulated data frame is re-modulated for using like the training mode. Fig. 2 shows the proposed method.

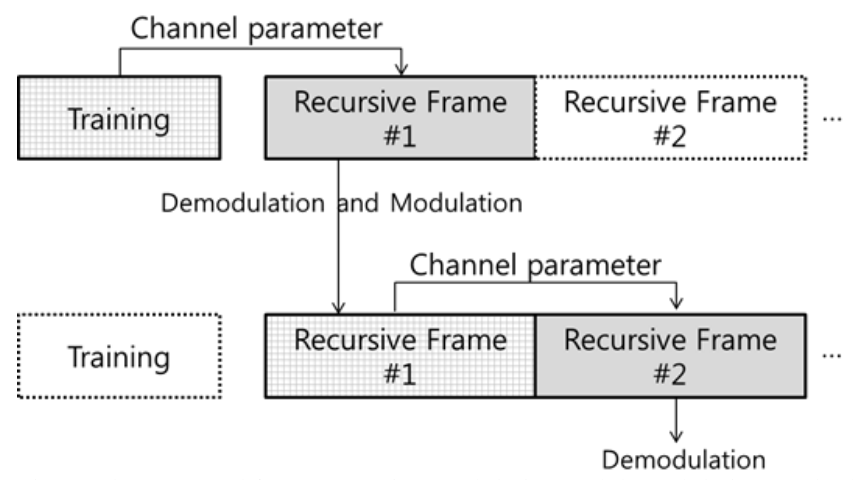

Fig. 2. The proposed frame recursive modulation and demodulation method

\section{B. Receiver Structure}

In this paper, we present the PSK receiver structure on Fig. 3. The desired signal (or training sequence) consists of correlator-banks with different Doppler-shifted replicas of the transmitted waveform. After estimation and recovery the Doppler shift frequency using the correlator-banks, the received signal is passed through the matched filter and sampled to symbol interval. The symbol spaced discrete signal is conducted second Doppler frequency recovery based on Kay's estimator. The distorted phase in signal is recovered by PLL(phase lock loop). Then, the equalizer compensates the signal distortion caused by multipath propagations. We used the DFE(decision feedback equalizer) based on RLS(recursive least square) algorithm [11]. The output of the equalizer is finally receiver output symbol and is used for frequency estimation and input of equalizer like desired signal. The buffers need to save the recursive frame for a moment exist at the receiver input and the output.

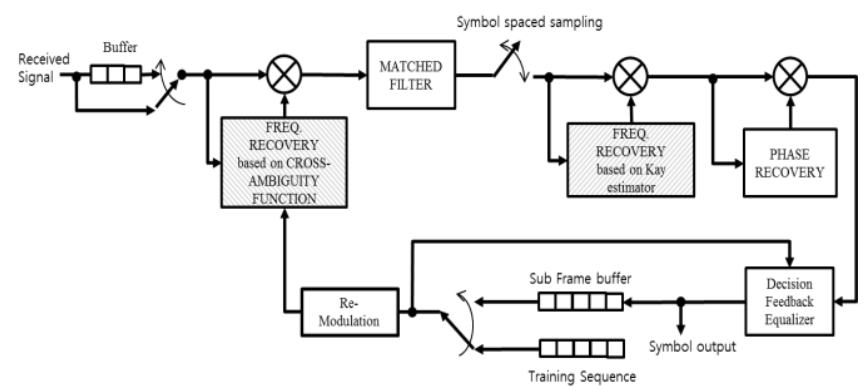

Fig. 3. Receiver structure

\section{Simulation RESUlts}

Simulation was performed to evaluate the performance of 
the proposed method. Table I shows the simulation environment parameters. It is assumed that the channel has the Rayleigh fading and white Gaussian noise. We also assumed the Doppler effect by movement. The Doppler parameter is dependent on carrier frequency and relative speed. Therefore, we present the Doppler term as the Doppler factor.

$$
\text { Doppler factor }=\frac{f_{s}}{f_{o}}
$$

$f_{s}$ : source frequency, $f_{o}$ : observed frequency

TABLE I: SIMULATION PARAMETERS

\begin{tabular}{lc}
\hline \hline Parameters & Values \\
\hline Modulation & QPSK (quadrature phase shift keying) \\
Data length & 1,000 symbols \\
Training sequence length & 256 symbols \\
Recursive frame length & $50,100,250,500$ symbols \\
Fading & Rayleigh fading \\
SNR & $5 \sim 25 \mathrm{~dB}$ \\
Doppler factor & $1 \sim 1.00014$ \\
\hline \hline
\end{tabular}

Because of channel randomness, we executed same simulations for ten times and calculated on average uncoded BER (bit error rate) value when channel coding is not used. The performance of the conventional communication system is shown on Fig. 4. The communication performance became worse when the shift frequency increased and SNR reduced.

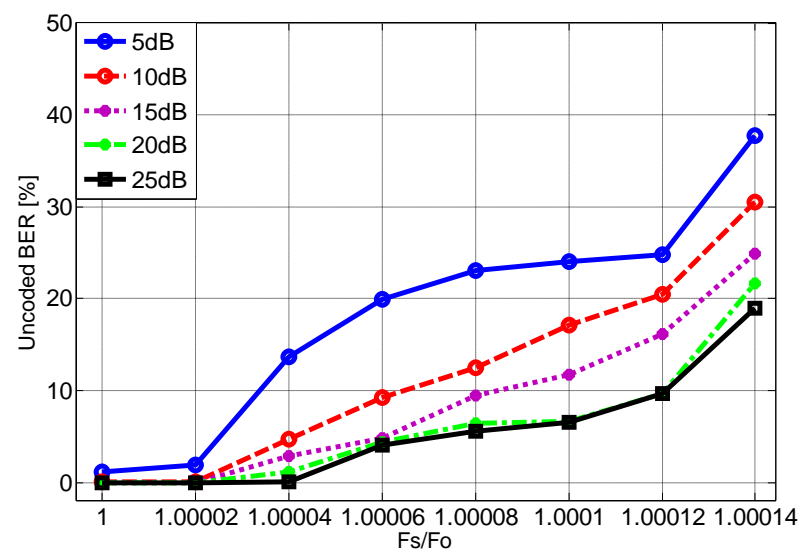

Fig. 4. Performance of the conventional communication packet system.

We compared the performance of the proposed method with conventional system. Fig. 5 shows the performance by recursive length when SNR is 5 and $25 \mathrm{~dB}$. When SNR was 5 $\mathrm{dB}$, the proposed method's performance was similar to or worse than conventional packet system. Because the error at recursive data frame in the head of data packet leaded to wrong decision. This error was easily occurred when SNR decreased and the Doppler shift frequency increased. And the influence of error in the head of data packet was increased at next recursive frame. This is the error propagation phenomenon. Where the channel environment with high SNR, the performances of the proposed method were better than conventional method except recursive frame length 500 symbols. Especially, the shorter recursive frame length was, the better performance had. We confirmed that the communication performance was improved maximum $17 \%$ by the proposed method.
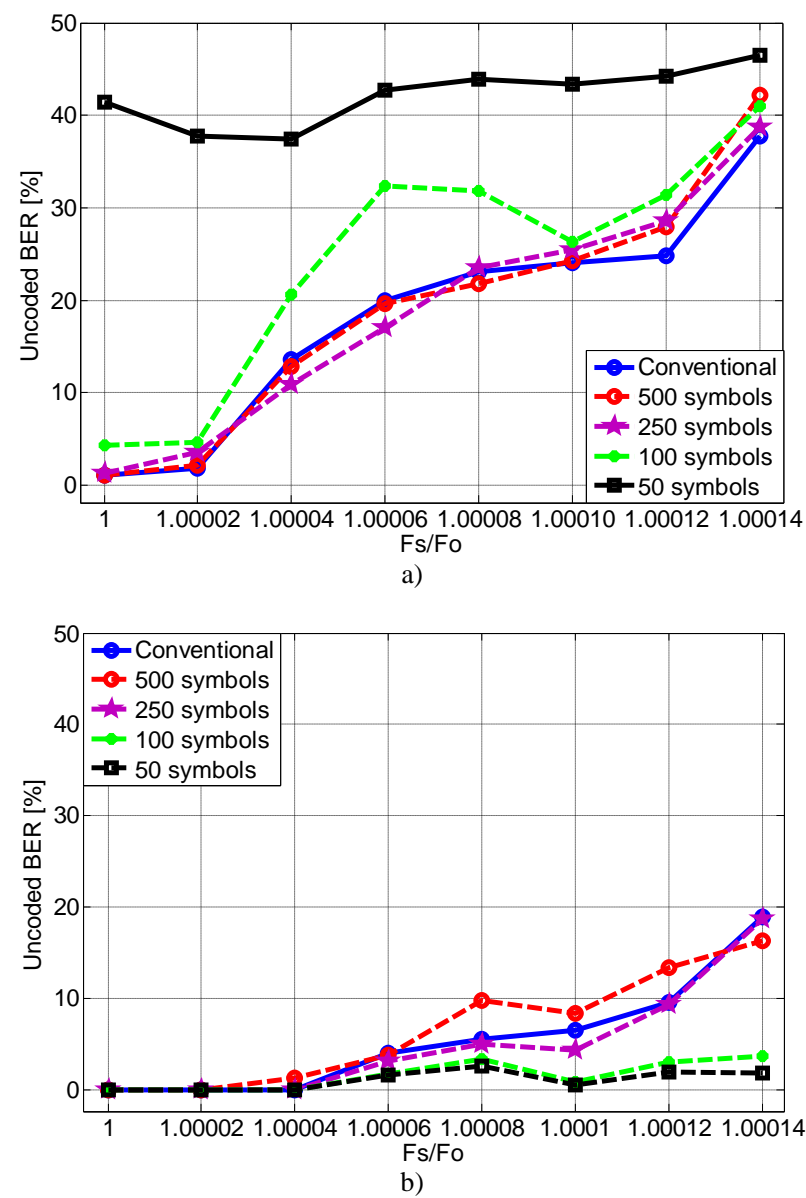

Fig. 5. Comparison of the performance by recursive frame length, a) SNR $5 \mathrm{~dB}$, b) SNR $25 \mathrm{~dB}$.

\section{CONCLUSIONS}

In wireless communication, the Doppler effect by movement decreases the transmission efficiency and distorts the transmitted signal. The conventional communication packet system of which data frame is only demodulated using the estimated channel parameters in training mode is sensitive to time-variance of channel. In this paper, we proposed the frame recursive modulation and demodulation method that is able to estimate the time-varying channel parameter during the data for increasing transmission efficiency and reducing the distortion caused by channel. We compared the performance of the proposed method with conventional method in the simulation environment with the Rayleigh fading and the Doppler effect. In the fading channel with low SNR and large Doppler shift frequency, the uncoded BER of the proposed method was similar to or larger than conventional method. Because the error bits in first recursive frame leaded to wrong decision at next recursive frame. In the channel with high SNR, the communication performance was improved maximum $17 \%$ by the proposed method. We confirmed that the proposed method has good performance in high SNR and short recursive frame length.

\section{ACKNOWLEDGMENT}

This work was supported by Ministry of Education and National Research Foundation of Korea (NRF) through the Human Resource Training Project for Regional Innovation and by Agency for Defense Development under the Contract UD130007DD. 


\section{REFERENCES}

[1] M. Katayama, A. Ogawa, and N. Morinaga, "Carrier synchronization under doppler shift of the nongeostationary satellite communication system," in Proc. ICCS/ISITA '92, Singapore, Nov. 1992, pp. 466-470.

[2] C. Tang, B. Lian, and L. Zhang, "Doppler-aided channel estimation in satellite communication base on frequency-domain equalization," in Proc. ICNS '2013, Herndon, VA, Apr. 2013, pp. 1-5.

[3] D. Tse and P. Viswanath, Fundamentals of Wireless Communication, Cambridge University Press, ch. 2, 2005.

[4] B. S. Sharif, J. Neasham, O. R. Hinton, and A. E. Adams, "Computationally efficient doppler compensation system for underwater acoustic communications," IEEE J. Oceanic Eng., vol. 25, no. 1, pp. 52-61, Jan. 2000.

[5] J. Rosen and L. Q. Gothard, Encyclopedia of Physical Science, Facts on File, 2009, p. 155.

[6] A. W. Rihaczek, Principles of High-Resolution Radar, Peninsula Publishing, ch. 3, 1985.

[7] S. Tao, "An improved frequency estimator for synchronization of PSK signals with large frequency offset," in Proc. Int. Conf. Wirel. Commun., Netw. Mob. Comput. (WiCOM '08), Dalian, China, Oct. 2008, pp. 1-3.

[8] J. K. Cavers, "An analysis of pilot symbol assisted modulation for Rayleigh fading channels," IEEE Trans. Vehicular Tech., vol. 40, pp. 686-693, Nov. 1991.

[9] S. Sampei and T. Sunaga, "Rayleigh fading compensation for QAM in land mobile radio communications," IEEE Trans. Vehicular Tech., vol. 42, pp. 137-147, May 1993.

[10] H. Li, Y. Iwanami, and T. Ikeda, "Symbol error rate analysis for MPSK under Rician fading channels with fading compensation based on time correlation," IEEE Trans. Vehicular Tech., vol. 44, pp. 535-542, Aug. 1995.

[11] W. Zhuang, "RLS algorithm with variable forgetting factor for decision feedback equalizer over time-variant fading channels," Wireless Personal Communication, vol. 8, pp. 15-29, Aug. 1998.

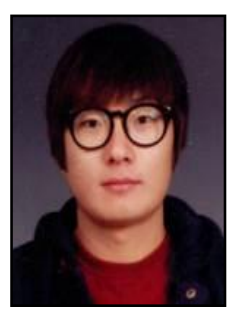

Chan-Ho Hwang was born in Republic of Korea. He received his B.S. degree in the Dept. of Radio Communication Engineering, Korea Maritime and Ocean University, Busan, Republic of Korea, in 2013. Currently, he is working on a M.S. degree in the Dept. of Radio Communication Engineering, Korea Maritime and Ocean University, Busan, Republic of Korea. His research interests include underwater communication and acoustic signal processing.

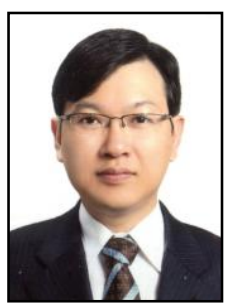

Ki-Man Kim received his B.S. degree in electronic engineering, Yonsei University, Seoul, Republic of Korea, in 1988. And he obtained his M.S. and PhD degrees from Electronic Engineering, Yonsei University, in 1990 and 1995, respectively. Currently, he is a professor at the Dept. of Radio Communication Engineering, Korea Maritime and Ocean University, Busan, Republic of Korea. His research interests include underwater communication, SONAR (sound navigation and ranging) signal processing, and array signal processing. 